\section{EARLY UNIVERSE}

\section{Back to the beginning Astrophys. J. 819, 129 (2016)}

After 26 years in the business, the Hubble Space Telescope has surprised astronomers once again. This time, Pascal Oesch and collaborators managed to observe a bright galaxy (GN-z11) in its infancy, some 13.4 billion years ago - or 400 million years after the Big Bang. In other words, this galaxy represents the farthest one that we know. In stellar evolutionary terms, GN-z11 formed 200-300 million years after the first stars appeared.

Combining the Hubble images with those taken by the Spitzer Space Telescope, an infrared observatory, reveals that the old galaxy is 25 times smaller than the Milky Way but is forming stars at 20 times the rate of our galaxy. The high rate of activity provides clues to the formation of the early Universe. However, this distance is the limit of Hubble's range. We must rely on future observations by the James Webb Space Telescope (set to launch in 2018) or the Wide-Field Infrared Survey Telescope (mid-2020s) to learn more about the birth of the earliest stars.

\section{TRAFFIC NETWORKS}

\section{Back-street ploys}

Nature Commun. 7, 10793 (2016)

We've all wasted time in a traffic jam imagining there might be a better way to get from A to B. But who among us has actually sat down and done the calculation? Serdar Çolak and colleagues have now provided us with a systematic study showing that traffic congestion can be alleviated, simply by modifying the choice of route. By correlating the road networks of five different cities with mobile phone data, the authors were able to map travel demand with road supply - finding that the ratio could be used to account for the hours lost in congested traffic.

Their proposed strategy for congestion relief involves a centralized routing system that lends credence to the claim that taking the back streets can legitimately relieve traffic flow problems. Optimization techniques for reducing overall congestion could feasibly be incorporated into existing technologies for route finding. The only problem, the authors note, is that individual drivers are likely to find the time savings imperceptible, so choosing the alternative route would really constitute a vote for the common good.

\section{VALLEYTRONICS}

\section{Sound out}

Phys. Rev. Lett. 116, 093901 (2016)

Condensed-matter physicists are making advances that could allow for the information carrier of future electronic devices to be the electron's spin or valley index, rather than its charge. Although spin is an intrinsic property, valley refers to the dips in the electronic band structure that, when the electrons cannot easily scatter out, effectively introduce an additional degree of freedom. The valley index therefore emerges from the way that the electron waves travel through a crystal structure. Jiuyang Lu and colleagues have now shown how this concept can be extended to sound waves travelling through a sonic crystal.

Considering a hexagonal array of triangular steel rods immersed in water, Lu et al. predicted that the acoustic valley states would be vortex-like, having both chirality and orbital angular momentum. These states would be excitable using external
PHASE TRANSITIONS

Dimensionality matters
Nature http://doi.org/bdb4 (2016)

Before a crystal hits its melting temperature, a curious process of 'premelting' occurs: a thin liquid layer develops at the crystal's surface. To better understand this process, Bo Li and colleagues have studied it in monolayers and bilayers of colloidal crystals - the advantage being that individual particles can be relatively easily monitored via optical microscopy methods.

Colloids with attractive interactions were required for preparing solid-vapour interfaces. The authors used poly (methyl methacrylate) spheres with a diameter of $2.02 \mu \mathrm{m}$, arranged in triangular lattices sandwiched between two glass plates. The interaction strength between the colloids was tuned by varying the temperature between 20 and $30^{\circ} \mathrm{C}$. Counterintuitively, (pre)melting was induced by lowering the temperature, as this reduced the attraction.

For the monolayer, incomplete premelting with a constant liquid-layer thickness was observed, as well as - at the onset of premelting - an isostructural solid-solid phase transition in the 'bulk' of the 2D crystal. Such a phase transition was not seen for the bilayer. Instead, conventional melting was obtained, with the surface-liquid's thickness diverging when reaching the melting point. sounds, but would adhere to selection rules, providing a method for selectively creating acoustic vortices with controllable chirality. Such systems may even exhibit acoustic analogues of Hall effects. So although scalar acoustics lacks an equivalent to spin, the principles of valleytronics provide additional tools for manipulating sound waves.

\section{BIOACOUSTIC MONITORING}

\section{A whale of a job}

Phys. Rev. E 93, 022138 (2016)

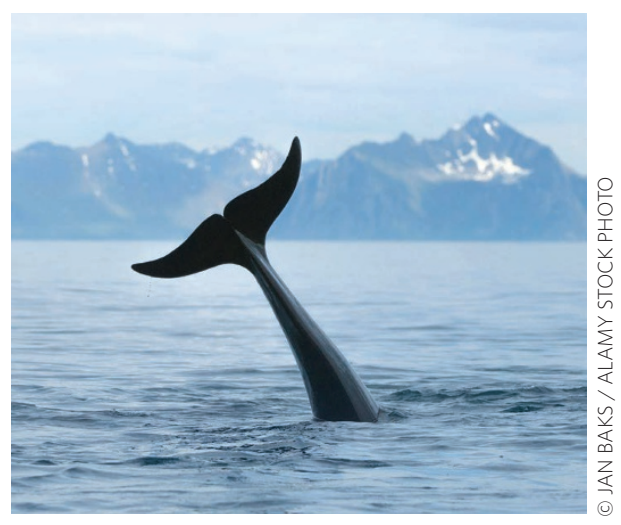

Monitoring the fascinating sounds made by whales - or any other species of animal in the wild, for that matter — is made challenging by the fact that recordings typically lack information regarding the sender and its context. And given the different sensoryprocessing systems of these animals, is it even meaningful to categorize single calls according to features that seem relevant to human observers?

Heike Vester and colleagues have opted for a completely different approach. Instead of separating and sorting vocalizations into types, they compared ensembles of sounds produced by different groups of long-finned pilot whales recorded in northern Norway. They achieved this by performing a cepstral decomposition of the noise signal, a process akin to a spectral decomposition. Computing and analysing the distribution of the resulting cepstral coefficients allowed the authors to identify different whale groups in a statistically significant way.

Taking their cue from machine learning, where ensembles are sometimes referred to as bags, Vester and co-workers termed this the bags-of-calls-coefficients approach. One might say the results of this approach speak for themselves: the authors uncovered differences between social groups of whales, consistent with the existence of distinct vocal cultures and dialects among the cetaceans.

Written by May Chiao, Luke Fleet, Abigail Klopper, Andrea Taroni and Bart Verberck. 